\title{
Cochlear Implantation Using a Suprameatal Approach in a Case of Severely Contracted Mastoid Cavity
}

\author{
Ji Eun Choi, Jeon Yeob Jang, and Yang-Sun Cho \\ Department of Otorhinolaryngology-Head and Neck Surgery, Samsung Medical Center, Sungkyunkwan University School of Medicine, \\ Seoul, Korea
}

Received May 20, 2014

Revised July 20, 2014

Accepted September 3, 2014

Address for correspondence

Yang-Sun Cho, MD, $\mathrm{PhD}$

Department of Otorhinolaryngology-

Head and Neck Surgery,

Samsung Medical Center,

Sungkyunkwan University

School of Medicine,

81 Irwon-ro, Gangnam-gu,

Seoul 135-710, Korea

Tel $+82-2-3410-3578$

Fax $+82-2-3410-3879$

E-mailyscho@skku.edu
Although cochlear implantation using posterior tympanotomy has been performed worldwide, other alternative approaches might be more beneficial and convenient in some selected cases. Of these, suprameatal approach was reported to be one of useful options in cases with narrow facial recess, anteriorly located facial nerve and an ossified cochlea. We describe a case of cochlear implantation using the modified suprameatal approach in a severely contracted mastoid cavity and suggest another indication of this approach. Korean J Audiol 2014;18(3):144-147

KEY WORDS: Cochlear implantation · Suprameatal approach · Mastoid.

\section{Introduction}

Cochlear implantation is generally performed by the way of mastoidectomy with posterior tympanotomy and cochleostomy or round window approach followed by insertion of electrode into the cochlea. ${ }^{1)}$ Although this surgical method may be relatively easily performed, alternative methods were introduced to reduce the surgical risk such as facial nerve palsy. ${ }^{2-5)}$ Among those, the suprameatal approach (SMA) was developed as one of the alternatives to the conventional approach in 1999 by Kronenberg. ${ }^{4,67)}$ The middle ear is exposed from the external auditory canal (EAC), providing wide exposure of the promontory and cochleostomy site. The electrode is introduced into the cochlea via suprameatal tunnel bypassing the mastoid cavity. ${ }^{4,-68}$

The SMA has some concerns regarding the angle of insertion into the scala tympani because there is no direct vision into the scala tympani and the possibility of inducing electrode

This is an Open Access article distributed under the terms of the Creative Commons Attribution Non-Commercial License (http://creativecommons. org/licenses/by-nc/3.0/) which permits unrestricted non-commercial use, distribution, and reproduction in any medium, provided the original work is properly cited. insertion trauma. ${ }^{9,10)}$ Although considering of these disadvantages, SMA has indications in narrow facial recess, an anteriorly located facial nerve and an ossified cochlea ${ }^{8}{ }^{8}$ We have experienced a case of cochlear implantation using this approach in a case with severely contracted mastoid cavity and suggest an additional indication of SMA in the procedure of cochlear implantation.

\section{Case Report}

A 13-year-old-girl was referred to our clinic for evaluation of candidacy for a cochlear implantation. Her parents found that her hearing was impaired when she was 1 year old and bilateral sensorineural hearing loss was confirmed. Since then, she has been wearing bilateral hearing aids (Sumo DM, Oticon, Denmark), and her hearing loss progressed thereafter. Past medical history was not noticeable except for the history of bilateral strabismus surgery, and there was no family history of hearing loss. Physical examination revealed normal tympanic membranes (TM) at both sides, and pure tone audiogram showed bilateral profound hearing loss of about $95 \mathrm{~dB}$. Additional audiologic tests including auditory brainstem response and oto- 
acoustic emission showed no responses to test stimulus. The open-set sentence recognition score was $6 \%$ and speech perception test using sentence materials showed marked limitations of daily conversation without visual cue that was applicable to categories of auditory performance (CAP) score 2 (response to speech sound). There was no evidence of inner ear anomaly in the imaging studies including magnetic resonance imaging and computed tomography of temporal bone (TBCT). Bilateral prominent sigmoid sinus with contracted mastoid cavity was observed in the TBCT (Fig. 1). As both sides of cochlea were radiologically intact, mastoids were sclerotic and the auditory functions were similar, cochlear implantation was planned on the right side for easy manipulation. Although the presence of a prominent sigmoid sinus was noted preoperatively, it was not considered to be a obstacle for conventional approach. However, during the operation, posterior tympanotomy could not be made due to the prominent sigmoid sinus and only a small antrostomy could be made. Instead of trying to make a posterior tympanotomy, we used a modified SMA. After making a well for the receiver-stimulator, a tympanomeatal flap was elevated and a cochleostomy site was made at the promontory antero-inferiorly to the round window. The electrodes array (Harmony, Advanced Bionics Corp., Valencia, CA, USA) were introduced to the mastoid antrum, lateral to the short process of incus, to the middle ear cavity and the fulllength was successfully inserted into the cochleostomy site (Fig. 2A-C). Intraoperative radiography showed well-positioned electrodes at the right cochlea (Fig. 2D) and the results of intraoperative impedance test and neural response imaging were successful for all 16 electrodes. The tympanomeatal

Fig. 1. Preoperative temporal bone computed tomography showed prominent sigmoid sinus (arrows) causing contracted mastoid cavity. A: Axial view. B: Coronal view.
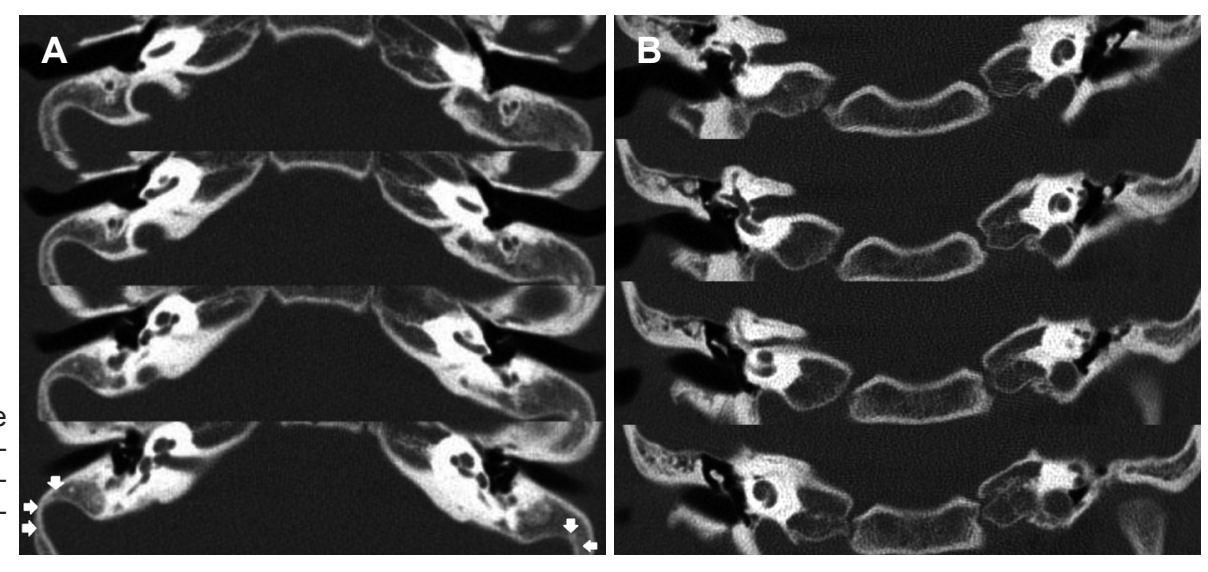

Fig. 2. Intraoperative findings (right ear). A: Prominent sigmoid sinus was observed in a contracted mastoid cavity (asterisk). B: Cochleostomy was made via external auditory canal. C: Electrode was introduced through the antrum and full insertion was made. D: Postoperative transorbital view shows correct positioning of the electrode array in the right cochlea.
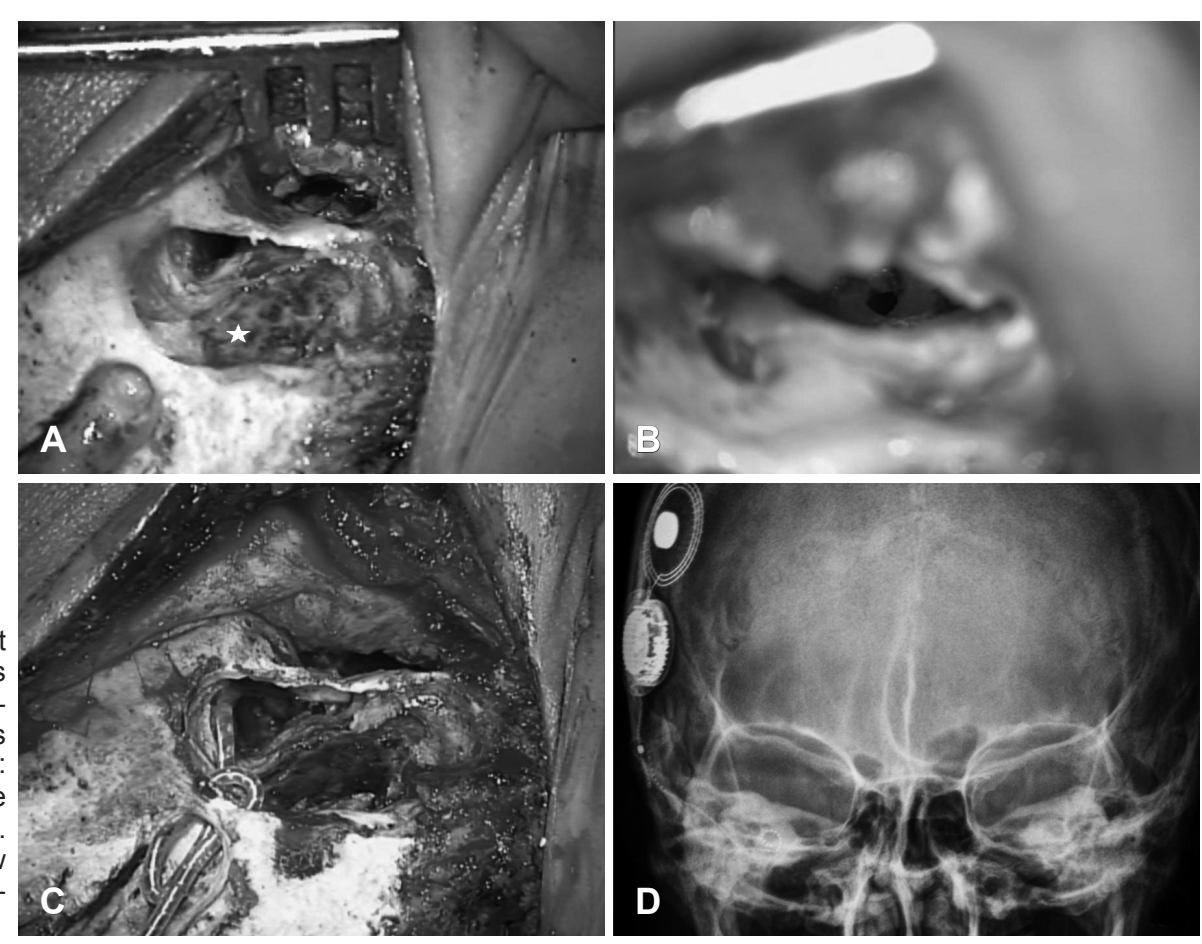

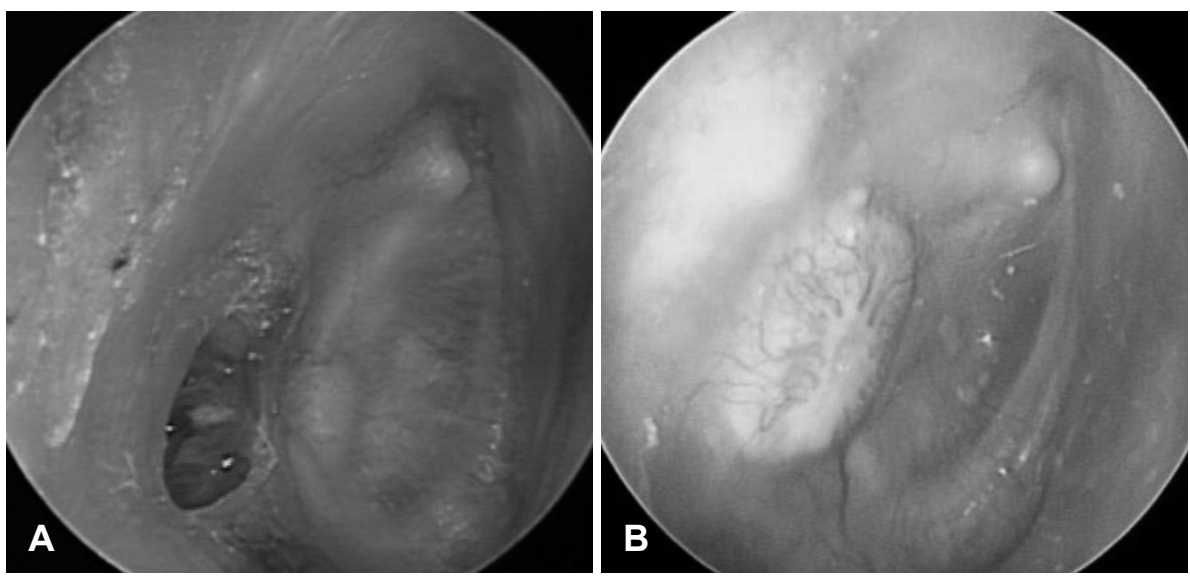

Fig. 3. TM findings with a small posterior perforation 2 months postoperatively. A: The electrode was visible posterior to the perforation. B: Wellhealed TM after 1 month later of tymapnoaplsty with cartilage reinforcement. TM: tympanic membranes.

flap was placed back, and EAC was packed with small pieces of gelfoam. Gelfoam was removed at 3 weeks postoperatively, and the TM was well-healed at that time.

However, at 2 months of follow-up, a small perforation was found at the posterior part of TM without a sign of infection, and the electrode was observed to be in contact with the TM (Fig. 3A). Subsequently, transcanal tympanoplasty was performed with cartilage reinforcement to cover the electrode. The TM was healed uneventfully (Fig. 3B), and maintained without trouble. The CAP score improved to 5 (understand common phrases, no lip-reading) and open-set sentence recognition score improved to $90 \%$ at 2 years postoperatively.

\section{Discussion}

Conventional technique using mastoidectomy with posterior tympanotomy has been performed worldwide for cochlear implantation since its first introduction in 1961 by House. ${ }^{1)}$ However, drilling through the facial recess, bordered posteriorly by the mastoid segment of the facial nerve and anteriorly by the chorda tympani, can endanger the facial nerve and the chorda tympani. ${ }^{4}$ Due to the risk of facial nerve palsy, although being rare and temporary, alternative surgical methods have been introduced so far. ${ }^{2,3,5)}$ Among these, the suprameatal approach eliminates the need for mastoidectomy and posterior tympanotomy which is performed in a safe distance from the facial nerve, and shortens the surgical time. ${ }^{4,6-8,11)}$ The middle ear is exposed through a postauricular incision and elevation of the posterior skin of EAC. The direct access to the middle ear provides a wider exposure of the promontory and better localization of the cochleostomy site. , $^{67,10,11)}$

In spite of these advantages, SMA also has several drawbacks. Recently, many surgeons attempt to reduce the risk of damage to the interior structure of the cochlea. But it is difficult to observe the procedure of insertion along the basal turn in SMA because the axis of visualization is different to the course of insertion. ${ }^{10)}$ Furthermore, a round window approach is difficult to perform with this approach. ${ }^{11)}$ Another concern is the relatively steep insertion of the electrode, which increases the risk of kinking and rotation of the electrode. This may possibly lead to ruptures of the basal membrane and a loss of residual hearing. ${ }^{10)}$ However, despite these assumptions, many studies report the electrode insertion process to be smooth without resistance. ${ }^{4,8)}$

SMA could be one of the useful options in case of narrow facial recess, an anteriorly located facial nerve and an ossified cochlea. ${ }^{8)}$ The wider exposure of the promontory enables easier manipulation of the surgical instruments, which makes this approach more suitable for cases with ossified cochlea that require extensive drilling of cochlear duct. ${ }^{7)}$ Another indication of SMA is patients with CHARGE syndrome which is characterized by choanal atresia, colobomatous microphthalmia, heart disease, hearing loss, and mental retardation. Many CHARGE syndromes have anatomic malformations including aberrant course of the facial nerve and such anomalies make the surgical procedures more challenging and dangerous. ${ }^{12)}$ The SMA is safer when important surgical landmarks (i.e., lateral semicircular canal and incus) are absent or aberrant course of the facial nerve is encountered. ${ }^{12)}$ Additionally, as in the presenting case, SMA can be useful in cases with severely-contracted mastoid cavity where posterior tympanotomy could not be made. Instead, the round window could be accessed through the antrum and the tympanic cavity.

The original SMA employs a small hole at the superior wall of EAC without mastoidectomy for the route of electrode. ${ }^{7)}$ However, in this case, electrode was entered through antrostomy which is similar to modification of SMA by Postelmans, et al. ${ }^{11)}$ This modification could be performed in cases of a lowlying dura which presents an obstacle to the creation of the suprameatal tunnel.

As observed in the presenting case, if the electrode array contacts the TM after the insertion, perforation may easily oc- 
cur. Therefore, it is recommended to cover the posterior part of TM with autologous cartilage to prevent the complication. In summary, the SMA is one of the useful surgical methods that can be used alternatively in cases where the conventional posterior tympanotomy approach is difficult to be performed. The indication of this method may include narrow facial recess, anteriorly located facial nerve, ossified cochlea and severely-contracted mastoid. It would be helpful for cochlear implant surgeons to get familiar with this approach.

\section{REFERENCES}

1) House WF. Cochlear implants. Ann Otol Rhinol Laryngol 1976;85 suppl 27(3Pt2):1-93.

2) Colletti V, Fiorino FG, Carner M, Pacini L. Basal turn cochleostomy via the middle fossa route for cochlear implant insertion. Am J Otol 1998;19:778-84.

3) Kiratzidis T. 'Veria operation': cochlear implantation without a mastoidectomy and a posterior tympanotomy. A new surgical technique. Adv Otorhinolaryngol 2000;57:127-30.

4) Kronenberg J, Baumgartner W, Migirov L, Dagan T, Hildesheimer M. The suprameatal approach: an alternative surgical approach to cochlear implantation. Otol Neurotol 2004;25:41-4; discussion 44-5.
5) Chouard CH, MacLeod P. Implantation of multiple intracochlear electrodes for rehabilitation of total deafness: preliminary report. Laryngoscope 1976;86:1743-51.

6) Kronenberg J, Migirov L, Dagan T. Suprameatal approach: new surgical approach for cochlear implantation. J Laryngol Otol 2001;115: 283-5.

7) Kronenberg J, Migirov L, Baumgartner WD. The suprameatal approach in cochlear implant surgery: our experience with 80 patients. ORL J Otorhinolaryngol Relat Spec 2002;64:403-5.

8) Yin S, Chen Z, Wu Y, Wang L, Zhang J, Zhou W, et al. Suprameatal approach for cochlear implantation in 45 Chinese children. Int J Pediatr Otorhinolaryngol 2008;72:397-403.

9) Postelmans JT, van Spronsen E, Grolman W, Stokroos RJ, Tange RA, Maré MJ, et al. An evaluation of preservation of residual hearing using the suprameatal approach for cochlear implantation: can this implantation technique be used for preservation of residual hearing? Laryngoscope 2011;121:1794-9.

10) Postelmans JT, Grolman W, Tange RA, Stokroos RJ. Comparison of two approaches to the surgical management of cochlear implantation. Laryngoscope 2009;119:1571-8.

11) Postelmans JT, Tange RA, Stokroos RJ, Grolman W. The suprameatal approach: a safe alternative surgical technique for cochlear implantation. Otol Neurotol 2010;31:196-203.

12) Ricci G, Trabalzini F, Faralli M, D’Ascanio L, Cristi C, Molini E. Cochlear implantation in children with "CHARGE syndrome": surgical options and outcomes. Eur Arch Otorhinolaryngol 2014;271:489-93. 